\title{
In-Process Modal Parameter Identification for Spindle Health Monitoring
}

\author{
Ruqiang Yan ${ }^{1}$, Robert X. Gao ${ }^{2}$, and Li Zhang ${ }^{3}$ \\ ${ }^{1}$ School of Instrument Science and Engineering \\ Southeast University, Nanjing, 210096 CHINA \\ Email: ruqiang@seu.edu.cn \\ ${ }^{2}$ Department of Mechanical Engineering \\ University of Connecticut, Storrs, CT 06269 \\ Email:rgao@engr.uconn.edu \\ ${ }^{3}$ Global Research Center \\ General Electric Corporation, Niskayuna, NY 12309
}

\begin{abstract}
This paper presents a stochastic subspace identification (SSI)-based approach for in-process spindle health monitoring. The approach identifies modal parameters of a spindle from the structural excitations experienced by the spindle during its operation. Experimental results obtained from the spindle system under different speeds and loading conditions confirm the effectiveness of the approach. The results further indicate that variations in the modal parameters identified during the experiments can serve as an indicator for the spindle's health condition. Therefore, the SSI-based modal parameter identification method can be effectively utilized for monitoring spindle health condition.
\end{abstract}

Keywords: Spindle dynamics, non-intrusive testing, stochastic subspace identification, health monitoring 


\section{Introduction}

Spindles are essential elements in virtually all machine tools and their structural dynamic behavior directly affects the performance of machine tools. Identification of spindle modal parameters has been extensively investigated [1-6], and various analytical and experimental methods have been explored. For example, lumped mathematical models were applied to derive guidelines for improving the dynamics of machine tools [2]. Receptance coupling substructure analysis was used to predict the structural response of a spindle-holder assembly [3]. By combining receptance coupling analysis with beam theory [4], a spindle-holder assembly was modeled to predict chatter stability, based on the machine tool's Frequency Response Function (FRF). The FRF-based method was also developed to determine boundary conditions for stable metal removal rates in milling operation [5]. In another study [6], the impulse responses to hammer strikes on stationary spindles were analyzed to extract various parameters such as structural stiffness, damping, and dominant mode shapes. However, dynamics captured on a stationary spindle may not adequately represent the rotating nature of the spindle, since its modal parameters (e.g., natural frequency) may be affected by the actual operating conditions. For example, the natural frequency of a spindle was found to fluctuate with shaft speed and bearing preload [7]. Also, stiffer spring characteristics due to a higher axial bearing preload have been found to result in higher natural frequencies [8]. On the other hand, identifying such parameter changes during a spindle's operation can provide insight into the spindle's present working status and its future performance, as the spindle depredates due to wear and tear. Such insight can also help devise more effective and efficient signal processing algorithms, e.g. by selecting proper scales needed for signal decomposition using the wavelet transform [9].

While hammer-strikes have been widely used for identifying dynamic changes in a rotating spindle tool-tip for improved chatter stability prediction [10], a non-intrusive method is desirable to 
be compatible with the normal mode of spindle operation avoid potential damage to the machine tool. Experimental observations from a realistic, custom-built spindle test system have shown that a rotating spindle's response to instrumented hammer strikes is often overwhelmed by noise contamination. As a result, correlation with the input hammer strike is typically weak, as indicated by an average value of less than 0.5 in the coherence function (a measure of the degree of correlation) between each frequency component of the input and output signals (Figure 1b). Such low coherence values imply that a valid Frequency Response Function (FRF) of the spindle system is difficult to derive, thus affecting the accuracy of the identified mode parameters. This motivates the investigation of new techniques that improve modal parameter identification of a rotating spindle under changing operation conditions.

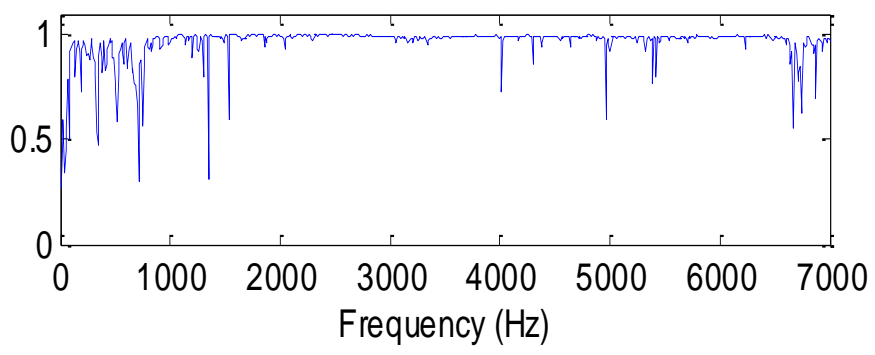

(a) Hammer strike on a stationary spindle

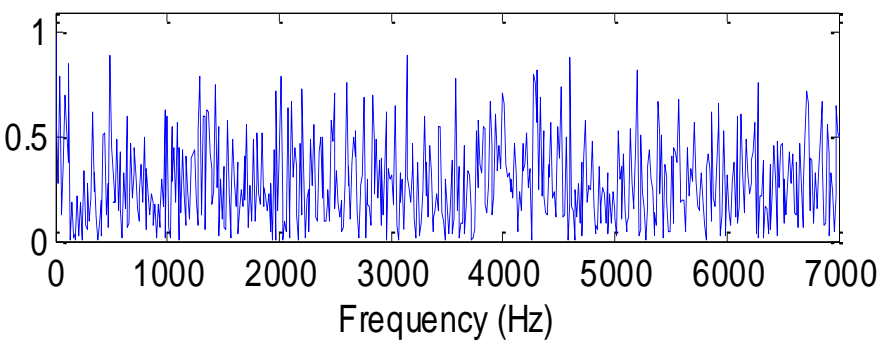

(b) Hammer strike on the same spindle rotating at 1,200 rpm

Figure 1. Coherence functions between hammer strikes and the spindle's structural response.

Output-only modal analysis has been studied in the area of civil engineering [11-17], due to the following advantages: (1) testing is relatively simple and fast, as no excitation equipment (e.g., vibration shaker or impact hammer) is needed; (2) testing does not interfere with the operation of the structure, thus enabling in-process monitoring; (3) measured response is representative of the 
real operating conditions of the structure; and (4) the method is capable of identifying the same modal parameters as the classical modal analysis, except for not being able to separate the mass and stiffness effect of the structure. Of the various techniques that have been explored for output-only modal analysis, such as natural excitation [11,12], stochastic subspace identification (SSI) [18], frequency domain decomposition [19], and random decrement [20], SSI has attracted increasing attention, and its application has been extended from civil engineering [21, 22] to various domains, such as power system [23], aerospace [24-26] and mechanical engineering [27-33]. As an example, a recursive adaptive SSI method was proposed for real-time monitoring low frequency electromechanical modes in power systems [23]. The SSI was also applied to in-flight data measured on a helicopter to validate and update its ground test models [24]. In another study, SSI performed on operational vibration data measured on a laser cutting machine has revealed an additional mode of vibration with direct influence on the cutting results, which wasn't identified by the classical modal analysis [27]. With a harmonic modification SSI approach, the modal frequency of the offshore wind turbine structure under the strong harmonic interference caused by rotating blades can be identified with increased accuracy [32].

Motivated by prior research, this paper presents an SSI-based modal parameter identification approach for rotating spindle health monitoring under varying operation conditions. Instead of fitting an empirical model to the FRF of a spindle from artificial excitations, the spindle's measured vibration output was used to extract modal parameters [18, 24, 34]. This approach accounts for dynamic changes caused by the rotations of the spindle without the need for artificial excitations [14], and thus does not suffer from the inherently low signal-to-noise ratio typically associated with hammer-striking a rotating spindle as shown in Figure 1b. The rest of this paper is organized as follows. Section 2 introduces the theoretic formulation of the SSI, and its ability to identifying mode parameters of structures subject to general unknown force input is verified analytically. A simulated 
test of a three Degree-of-Freedom (DOF) system is then performed to evaluate the effectiveness of the SSI approach in modal parameter identification. The application of the presented approach to spindle health monitoring is subsequently conducted on a custom-designed spindle test system during an accelerated run-to-failure test in section 3, in which effects of the rotating speed and preload on the spindle dynamics are discussed. Finally, conclusions are drawn in section 4.

\section{Stochastic Subspace Identification}

Stochastic subspace identification is a time-domain technique for output-only modal analysis, and is formulated using state-space models. Assuming that the vibrational behavior of a continual mechanical system (such as a spindle) can be analytically approximated by that of an equivalent, multiple degree-of-freedom (MDOF) system (e.g. a series of mass-spring-dampers), and the structural response is linear and time-invariant, the corresponding discrete state-space model of the spindle can be expressed as:

$$
\left\{\begin{array}{l}
x_{k+1}=A x_{k}+B u_{k}+w_{k} \\
y_{k}=C x_{k}+D u_{k}+v_{k}
\end{array}\right.
$$

where $x_{k}=x(k \Delta t)$ is the discrete-time state vector, $u_{\mathrm{k}}$ is the structural input displacement vector resulting from a known excitation force, $y_{k}$ is the system response vector, $A$ is the state matrix, $B$ is the input matrix, $C$ is the output matrix, and $D$ is the direct transmission matrix. The two components, $w_{k}$ and $v_{k}$, represent the disturbance noise to the spindle and measurement noise due to sensor inaccuracy, respectively, and are stochastic in nature. The state space dimension $n$ is determined by the number of independent variables needed to describe the physical system, i.e. the number of mass-spring-dampers as the constituent elements for spindle modeling. Given that no known excitation force is applied to the spindle when performing the output-only model analysis, the term $u_{\mathrm{k}}$ would vanish, and the system is then represented by the stochastic state-space model as $[13,18]:$ 


$$
\left\{\begin{array}{l}
x_{k+1}=A x_{k}+w_{k} \\
y_{k}=C x_{k}+v_{k}
\end{array}\right.
$$

Equation (2) indicates that the new state of the spindle physical system, $x_{k+1}$, can be obtained by the sum of the state matrix $A(\mathrm{n} \times \mathrm{n})$ multiplied with the old state vector $x_{k}(\mathrm{n} \times 1)$ and the disturbance noise vector $w_{k}(\mathrm{n} \times 1)$. Thus the dynamics of the spindle is completely characterized by the state matrix $A$ and the modal parameters can be extracted from its eigenvalues. Also as shown in equation (2), the measured system response vector $y_{k}(m \times 1)$ contains the observable part of the state vector $C x_{k}(\mathrm{~m} \times 1)$ and the measurement noise vector $v_{k}(\mathrm{~m} \times 1)$, with $\mathrm{m}$ being the number of sensors used for the measurements.

In order to identify the state matrix $A$ from the measured system response (vibrations of the spindle), an optimal estimator of the state-space model must be obtained based on the measured system response. This requires that such measured system response must be a Gaussian stochastic process with zero mean, which leads to

$$
\left\{\begin{array}{l}
E\left[y_{k}\right]=0 \\
E\left[y_{k+i} y_{k}^{T}\right]=\Lambda_{i}
\end{array}\right.
$$

where $\Lambda_{i}$ is the output covariance matrix and it describes the Gaussian stochastic process, i.e., the measured system response. This means once the state space model with the covariance matrix $\Lambda_{i}$ can be estimated, it will completely describe the statistical properties of the measured system response. The corresponding estimator that can generate such a state space model is then considered as the optimal estimator. Furthermore, equation (3) implies that the input noise processes $w_{k}$ and $v_{k}$ are also zero-mean Gaussian $\left(E\left[w_{k}\right]=0\right.$ and $\left.E\left[v_{k}\right]=0\right)$, which are defined by the covariance matrices as:

$$
E\left[\left(\begin{array}{c}
w_{p} \\
v_{p}
\end{array}\right)\left(\begin{array}{ll}
w_{q}^{T} & v_{q}^{T}
\end{array}\right)\right]=\left(\begin{array}{ll}
Q & S \\
S^{T} & R
\end{array}\right) \delta_{p q}
$$


where $\delta_{p q}$ is the Kronecker delta function, and $Q, S$, and $R$ are the noise covariance matrices. Similarly, the state vector $x_{k}$ is also zero-mean $\left(E\left[x_{k}\right]=0\right)$ with the state covariance matrices and the updated state-output matrices defined using the noise covariance matrices as:

$$
\begin{aligned}
& E\left[x_{k+1} x_{k+1}^{T}\right]=\Sigma=A \Sigma A^{T}+Q \\
& E\left[x_{k+1} y_{k}^{T}\right]=G=A \Sigma A^{T}+S
\end{aligned}
$$

Based on the covariance matrices defined in equations (4) to (6), the output covariance matrices can be rewritten as:

$$
E\left[y_{k+i} y_{k}^{T}\right]= \begin{cases}\Lambda_{0}=C \Sigma C^{T}+R & i=0 \\ \Lambda_{i}=C A^{i-1} G & i \neq 0\end{cases}
$$

These covariance matrices are also called the system matrices because they describe the stochastic properties of the state-space systems. They are used for the estimation of the state-space model, and thus the identification of the state matrix $A$. Another system matrix needed for estimation is the extended observability matrix, which is defined as:

$$
O_{i} \equiv\left(\begin{array}{l}
C \\
C A \\
C A^{2} \\
\cdots \\
C A^{i-1}
\end{array}\right)
$$

Two types of algorithms are commonly employed for state-space model estimation: data-driven and covariance-driven algorithms [18]. For the presented study, a reference-based data-driven algorithm was investigated. A Kalman filter is employed for the optimal prediction of the state vector $x_{k+1}$ by making use of the chosen reference sensor measurements, which is denoted as $\hat{x}_{k+1}$. A Kalman filter state sequence can be formed by the various Kalman filter state estimates as:

$$
\hat{X}_{i} \equiv\left(\hat{x}_{i} \hat{x}_{i+1} \ldots \hat{x}_{i+j-1}\right)
$$


Once the Kalman filter state estimates are obtained, numerical techniques can be applied to estimate the state-space model, e.g. using the QR-factorization technique [18], which essentially projects the row space of the future outputs into the row space of the past outputs. The projection can be factorized as the product of the observability matrix and the Kalman filter state sequences:

$$
P_{i}=O_{i} \hat{X}_{i}
$$

Thus, the Kalman filter state sequence is expressed as:

$$
\left\{\begin{array}{l}
\hat{X}_{i}=O_{i}^{-1} P_{i} \\
\hat{X}_{i+1}=O_{i-1}^{-1} P_{i-1}
\end{array}\right.
$$

By applying equation (11) and extending equation (2), the state-space spindle model is then obtained in the form of a set of linear equations:

$$
\left(\begin{array}{c}
\hat{X}_{i+1} \\
Y_{i \mid i}
\end{array}\right)=\left(\begin{array}{l}
A \\
C
\end{array}\right) \hat{X}_{i}+\left(\begin{array}{l}
\rho_{w} \\
\rho_{v}
\end{array}\right)
$$

where $Y_{i \mid i}$ is the measured system responses written in the form of a block Hankel matrix and $\left(\rho_{w} \rho_{v}\right)^{T}$ are the residuals. Since the residuals are uncorrelated with $\hat{X}_{i}$, the system matrices $A$ and $C$ can be solved in equation (12) as:

$$
\left(\begin{array}{l}
A \\
C
\end{array}\right)=\left(\begin{array}{l}
\hat{X}_{i+1} \\
Y_{i \mid i}
\end{array}\right) \hat{X}_{i}^{-1}
$$

Since the dynamic behavior of the spindle is represented by the state matrix $A$, the eigenfrequencies and modal damping ratios can be obtained from the eigenvalues of state matrix $A$, using known decomposition techniques.

It should be noted that the SSI assumes white noise input. For applications where such an assumption doesn't apply and additional forcing input of unknown amplitude exists, the method needs to be modified. This can be achieved by taking the white noise input as a virtual load that 
generates a corresponding forcing input to the components of interest [35], e.g., the spindle, as illustrated in Figure 2. With this representation, the measured response contains information on dynamics of both the loading component and the components of interest, thus the corresponding identification process includes not only the vibration modes associated with the components of interest, but also the modes belong to the virtual loading component. However, the modal parameters of the components of interest are separable from those of the virtually excited modes of the loading component. This can be analytically derived as follows.

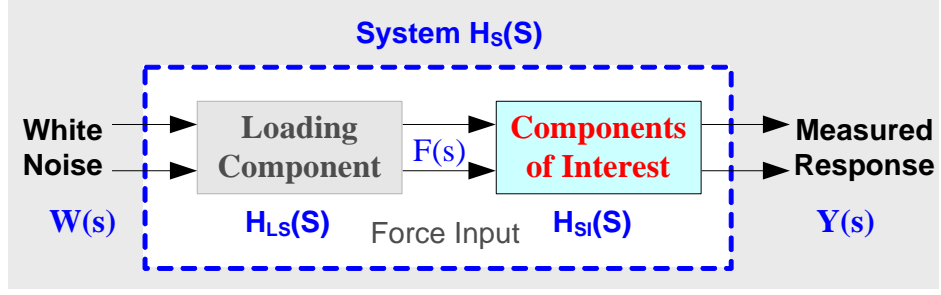

Figure 2 Illustration of output-only identification system

From the illustration in Figure 2, following relationship can be obtained:

$$
\left\{\begin{array}{l}
F(s)=W(S) \cdot H_{L S}(S) \\
Y(S)=F(S) \cdot H_{S I}(S)
\end{array}\right.
$$

where $W(S)$ denotes the white noise input, $F(S)$ is the force generated from the loading component applied to the components of interest. $Y(S)$ is the measured system response. $H_{L S}(S)$ and $H_{S I}(S)$ are the transfer function of the loading component and the components of interest, respectively. Substituting Eq. (14) into the transfer function $H_{S}(S)$ of the system, defined as:

$$
H_{S}(s)=Y(s) / W(s)
$$

following relationship can be established as the system's transfer function:

$$
H_{S}(s)=H_{L S}(s) \cdot H_{S I}(s)
$$

Assume there are $n$ and $m$ modes for the loading component and components of interest, respectively. For simplicity, the corresponding transfer function $H_{L S}(S)$ and $H_{S I}(S)$ can be written as: 


$$
\begin{aligned}
& H_{L S}(S)=\sum_{i=1}^{2 n} \frac{a_{i}}{s-\alpha_{i}} \\
& H_{S I}(S)=\sum_{j=1}^{2 m} \frac{b_{j}}{s-\beta_{j}}
\end{aligned}
$$

where $a_{i}, . \alpha_{i}, b_{j}$, and. $\beta_{j}$ are constants.

Substituting Eqs. (17) and (18) into equation (16) leads to:

$$
H_{s}(S)=\sum_{i=1}^{2 n} \frac{a_{i}}{s-\alpha_{i}} \sum_{j=1}^{2 m} \frac{b_{j}}{s-\beta_{j}}=\sum_{i=1}^{2 n} \frac{A a_{i}}{s-\alpha_{i}}+\sum_{j=1}^{2 m} \frac{B b_{j}}{s-\beta_{j}}
$$

where $A$ and $B$ are constants, and expressed as:

$$
\begin{aligned}
& A=\sum_{j=1}^{2 m} \frac{b_{j}}{\alpha_{i}-\beta_{j}} \\
& B=\sum_{i=1}^{2 n} \frac{a_{i}}{\beta_{j}-\alpha_{i}}
\end{aligned}
$$

Equation (19) indicates that all of the modal parameter of the components of interest can be identified using the SSI-based output-only modal analysis technique even though the requirement of white noise input is not satisfied. The only difference is that those modes related to the virtual loading component are also identified, but they can be differentiated and eliminated from the identification results based on human knowledge.

To evaluate the effectiveness of the SSI-based approach for modal parameter identification, a simulation model consisting of a three DOF spring-mass-damping system has been constructed. The system is shown in Figure 3, and the corresponding equation of motion is expressed in a matrix form as:

$$
M \ddot{X}(t)+C \dot{X}(t)+K X(t)=F(t)
$$


where $\ddot{X}(t), . \dot{X}(t)$, and $X(t)$ denote acceleration, velocity, and displacement matrices of the system response. The symbols $M, C$, and $K$ are mass, damping and stiffness matrices of the system.

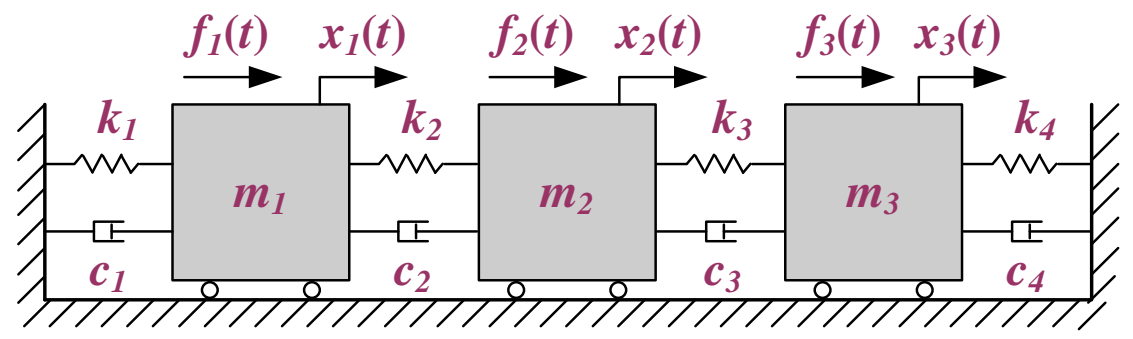

Figure 3. A three DOF spring-mass-damping system

For simplicity, the input force is equally applied to the three DOF spring-mass-damping system, i.e., $f_{1}(\mathrm{t})=f_{2}(\mathrm{t})=f_{3}(\mathrm{t})=f(\mathrm{t})$. A Gaussian white noise is used as input to a second-order single DOF system (working as the loading component shown in Figure 2) to generate such an input force. The transfer function of the single DOF system is expressed as:

$$
H_{L S}(S)=\frac{A_{L S}}{S^{2}+2 \xi_{f} \omega_{f} S+\omega_{f}^{2}}=\frac{25000}{S^{2}+2 \times 0.05 \times 628 \times S+628^{2}}
$$

The parameters of the three DOF spring-mass-damping system have been chosen as:

$$
\left\{\begin{array}{l}
m_{1}=2, m_{2}=3, m_{3}=4 \\
c_{1}=c_{2}=c_{3}=c_{4}=20 \\
k_{1}=k_{2}=k_{3}=k_{4}=40000
\end{array}\right.
$$

The corresponding nature frequencies of the system can then be theoretically calculated as 13.910, 25.990, and $36.419 \mathrm{~Hz}$, respectively. Figure 4 illustrates waveforms of the simulated input and output response $x_{l}(t)$ of the three DOF spring-mass-damping system. For accurate modal identification, data length that is much longer than the fundamental period is needed. Therefore, the data with about 1500 times the fundamental period of the first mode (i.e. 100s) is used in this study, and the waveform shown in Figure 4 is for purpose of illustration only. It is also noted that the output responses $x_{2}(t)$ and $x_{3}(t)$ of the system are similar to $x_{1}(t)$. 


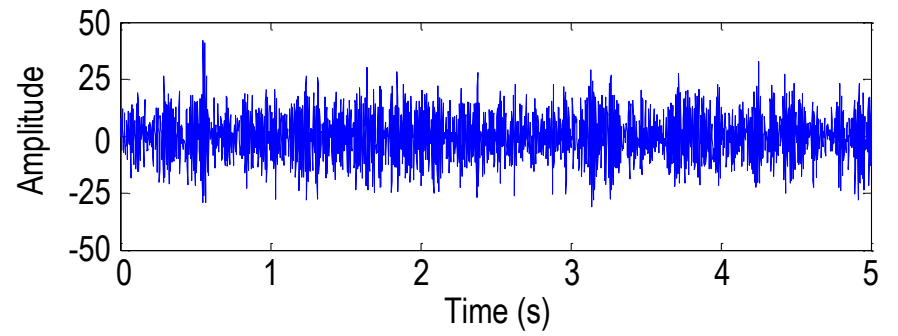

(a) Simulated input to the three DOF spring-mass-damping system

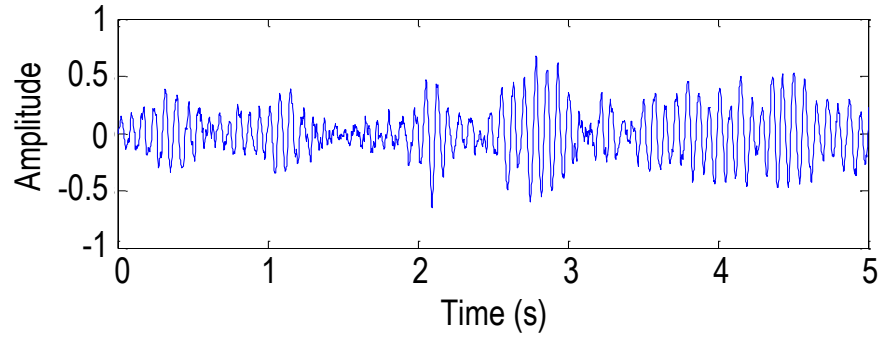

(b) Output respose $x_{I}(t)$ of the three DOF spring-mass-damping system

Figure 4. Waveforms of the simulated input and output response

The three responses $x_{1}(t), x_{2}(t)$ and $x_{3}(t)$ are used as input to the SSI algorithm for modal parameter identification. To ensure accurate modal parameter identification, the number of dimensions has been set in the algorithm as 40 initially, and the appropriate dimension is iteratively determined to extract the modal parameters. The estimated models are plotted in the stabilization diagram as shown in Figure 5. It is seen that the system modes appear "stable" across the various state space dimensions with the estimated modal parameters. As shown in Figure 5, there are four stable modes presented in the diagram, between $0 \mathrm{~Hz}$ and $150 \mathrm{~Hz}$. The modes (1), (2), and (3) correspond to the three DOF system while the mode (4) represents the assumed loading component, as indicated by Equation (23). The modal parameters can then be obtained by finding one state space dimension where all modes are stable. In this study, the natural frequencies of the three DOF system are estimated from the stabilization diagram, and the results are listed in Table I, which matches the theoretical values well. This verifies the effectiveness of the SSI-based approach for modal parameter identification. 


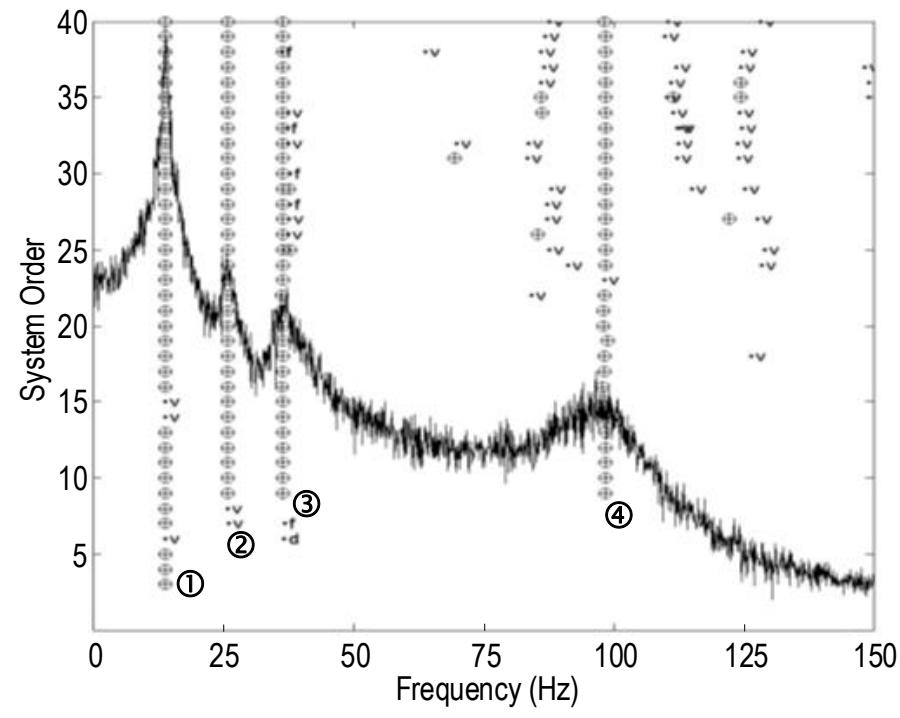

Figure 5. Stabilization diagram of the three DOF spring-mass-damping system

Table I. Comparison between theoretical and estimated natural frequencies

\begin{tabular}{|l|c|c|c|}
\hline Frequency Mode & (1) & (2) & (3) \\
\hline Theoretical (Hz) & 13.910 & 25.990 & 36.419 \\
\hline Estimated (Hz) & 13.918 & 25.928 & 36.428 \\
\hline Error (\%) & 0.05 & 0.24 & 0.02 \\
\hline
\end{tabular}

\section{Monitoring Operating Spindle}

To investigate the practical utility of the SSI-based approach for operating spindle health monitoring, experimental studies were conducted on a customized spindle test bed where the accelerometers are placed on the housing of the spindle, as shown in Figure 6. The test bed was supported by four $42 \mathrm{~mm}$-angular contact ball bearings mounted as duplex pairs on each end of the spindle shaft. An air cylinder applies a constant force to the spindle as the static preload. A dynamic load cylinder applies impulsive forces to the spindle shaft. The dynamic load cylinder serves two purposes in this study. First, it provides a means to accelerate the structural deterioration process of the spindle and establishes a spindle service life history. For testing the SSI-algorithm, the impulsive force was not used as input; only vibration data measured on the spindle were used for 
identifying modal parameters of the spindle, via a customized virtual instrument system [9]. Secondly, the impulsive force was used as input to conduct classical modal analysis, and both the impulsive force and corresponding spindle vibrations were measured and stored at the same time. The sampling frequency of the data acquisition board was set at $20 \mathrm{kHz}$, with the cut-off frequency of the anti-aliasing filter being $10 \mathrm{kHz}$.

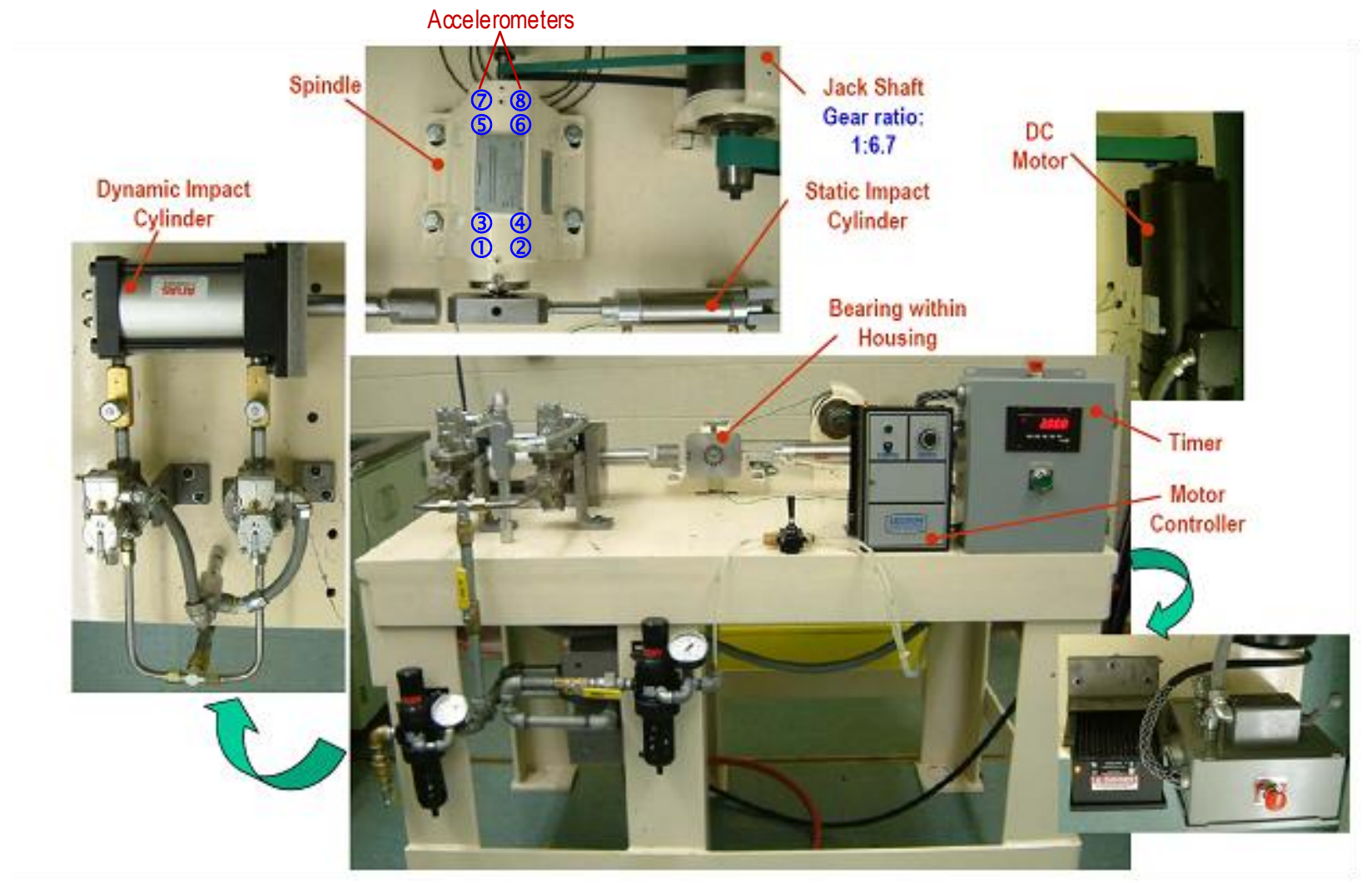

Figure 6. Spindle test bed setup

\subsection{Comparison with Classical Modal Analysis}

A comparison of the presented approach with classical modal analysis was first performed to establish an experimental basis for extracting the spindle modal parameters. As illustrated in the introduction section, using an instrumented hammer, strike-based test to the rotating spindle leads to weak correlation between the input and output signals due to contaminations from the spindle's structure-borne vibrations. To improve the signal-to-noise ratio (SNR), the dynamic load cylinder 
was utilized in this paper to provide stronger impulsive force inputs to the spindle than the hammer strikes. The coherence functions of cylinder strike on both stationary and rotating spindle were shown in Figure 7. As seen in Figure 7b, significantly improved correlation between the input force and output measurement of the rotating spindle was obtained as compared to that in Figure 1b, with the average coherence value approaching 1 . In addition, the correlation between the input force and output measurement of the stationary spindle has also been improved. As a result, air cylinder strikes were used to estimate the FRF of spindle in both the stationary and rotating conditions.

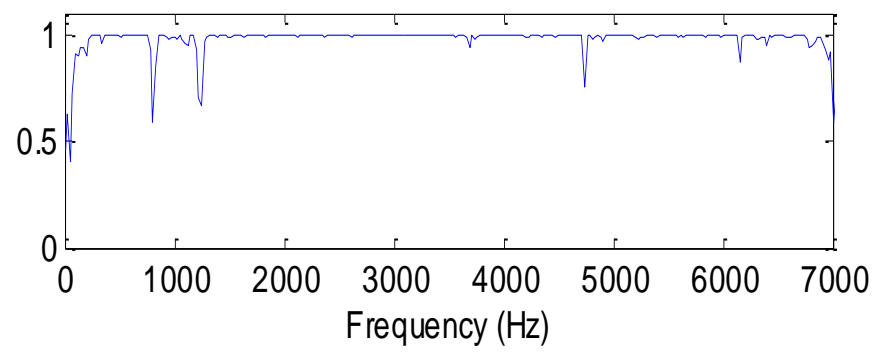

(a) Cylinder strike on stationary spindle

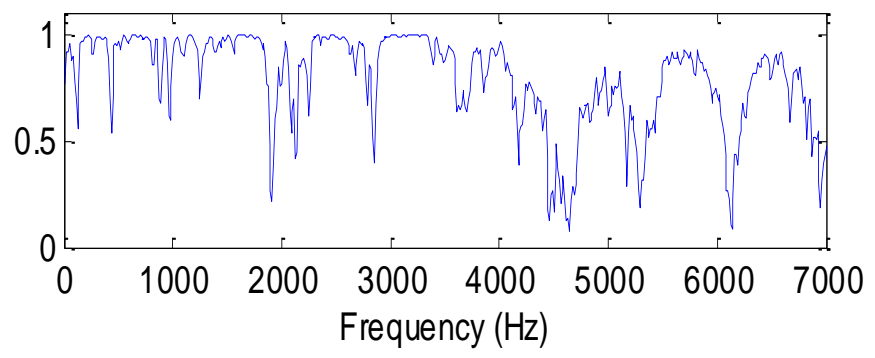

(b) Cylinder strike on rotating spindle

Figure 7. Coherence functions between cylinder strikes and the spindle's structural response.

The results of the FRF, which is estimated using the Rational Fraction Polynomials (RFP) method [36], are shown in Figure 8a and 8b, respectively. Three major structural modes (Figure 8a, denoted as (1), (2) and (4)) are identified for the stationery spindle in the frequency range from 2,000 $\mathrm{Hz}$ to $7,000 \mathrm{~Hz}$. In comparison, the spectrum for the rotating spindle revealed an additional mode at approximately $5,200 \mathrm{~Hz}$, denoted as (3) in Figure 8b. This indicates the potential drawback of classical modal analysis techniques in possibly missing certain dynamic characteristics of a rotating spindle, and illustrates the need for spindle dynamics identification under actual operating 
conditions. Figure $8 \mathrm{c}$ also shows the structural modes of the rotating spindle, however, with the SSI-based output-only modal analysis technique being employed. It is seen that the structural modes closely match those identified using cylinder strikes on the spindle (Figure $8 \mathrm{~b}$ ). Thus the applicability of the output-only modal identification technique is confirmed. Since the developed algorithm is performed while the spindle is rotating, it allows for in-process spindle health monitoring.

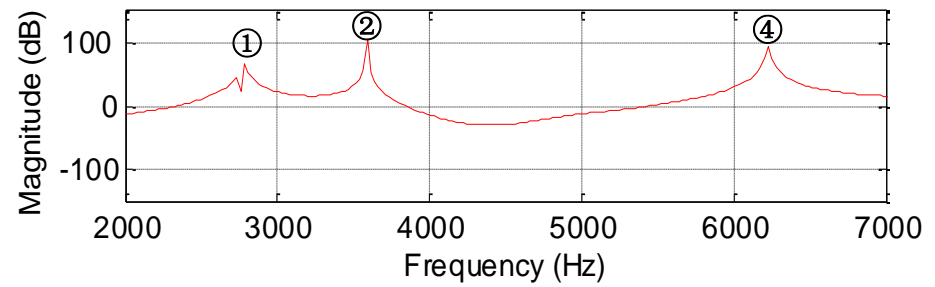

(a) Frequency response function using cylinder strike on stationary spindle

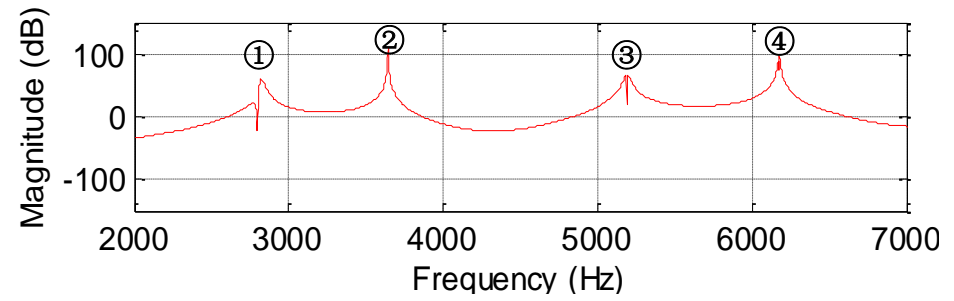

(b) Frequency response function using cylinder strike on rotating spindle

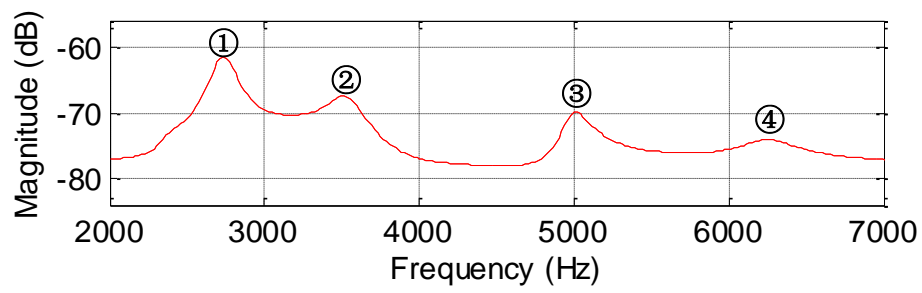

(c) Frequency response function using output-only measurement of rotating spindle

Figure 8. Comparison of modal parameters identified using classical and natural excitation methods

\subsection{Effects of Operating Conditions}

With the applicability of the SSI approach for operating spindle modal parameter identification being verified, further study in this paper is to apply this technique to in-process spindle health monitoring. To accelerate degradation of the spindle structure, dynamic impacts with amplitude of $13,300 \mathrm{~N}$ were applied to the spindle shaft rotating at $377 \mathrm{rad} / \mathrm{s}(3,600 \mathrm{rpm})$. The time interval for 
the two successive impacts was set to 1 second. While for SSI the input noise terms are usually assumed to be white, it was observed in preliminary experiments that the spindle dynamics also contains certain dominant frequency components, such as its rotational frequency and bearing characteristic frequencies. These frequency components cannot be separated from the eigenfrequencies of the system, and thus appear as poles of the state matrix A. Since the highest dominant frequency of the spindle (i.e., the ball passing frequency on the inner raceway, or BPFI) and its harmonics were found to be all below $2,000 \mathrm{~Hz}$ for the highest spindle rotational speed investigated $(837 \mathrm{rad} / \mathrm{s}$ or $8,400 \mathrm{rpm})$, they were removed from the data set through high-pass filtering so that only signals within the $2,000 \mathrm{~Hz}$ to $10,000 \mathrm{~Hz}$ (Nyquist) range were analyzed.

Using the SSI method, the natural frequencies and FRFs of the spindle were estimated for data sets collected under various operating conditions. Figure 9 shows a comparison of the estimated FRFs (red-colored solid lines) with the measured FRFs (blue, dotted lines) for the data sets where the speed was $126 \mathrm{rad} / \mathrm{s}(1,200 \mathrm{rpm})$ and no static load was applied. Clearly the dynamic behavior of the spindle has changed as a result of the accumulated impacts. Before the impact application when the spindle was "healthy", four structural modes (denoted (1) (2) (3) (4)) were identified, as shown in Figure 9a. As the number of impacts increased, two additional modes (denoted (5) (6) appeared in the frequency range of $8,000 \mathrm{~Hz}$ to $10,000 \mathrm{~Hz}$. As seen in Figure 10, the magnitude of the modes increased with the number of the impacts. In particular, mode (4) appears to have increased by about $15 \mathrm{~dB}$ after 1,100 impacts, as listed in Table II. Such an increase was also confirmed when the spindle output is analyzed by an alternative technique based on the wavelet envelope spectrum [37]. It can be seen in Figure 11 that vibration frequencies are concentrated at around $6,000 \mathrm{~Hz}$ in the wavelet domain, corresponding to mode (4) of the spindle. Furthermore, several frequencies associated with spindle unbalance $(100 \mathrm{~Hz}$ and $200 \mathrm{~Hz})$, bearing inner raceway defects $(664 \mathrm{~Hz})$, and the combined effect of spindle unbalance and inner raceway defects $(764 \mathrm{~Hz}$, etc.) were 
successfully detected, indicating the effects of the accumulated impacts. To further investigate the effect of the accumulated impacts on the spindle characteristics, Figure 12 shows the amplitude change on both the shaft frequency and the defect frequency components when the number of impacts increases. It can be seen that after a certain number of impacts, the amplitude of the defect frequency component starts to increase with the increased number of impacts, leading to the deterioration of the spindle condition. This increasing trend clearly verifies the effectiveness of the SSI-based approach for spindle health monitoring.

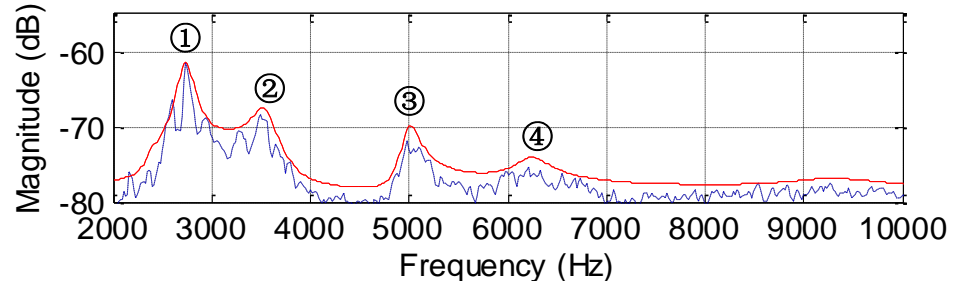

(a) Before impact test

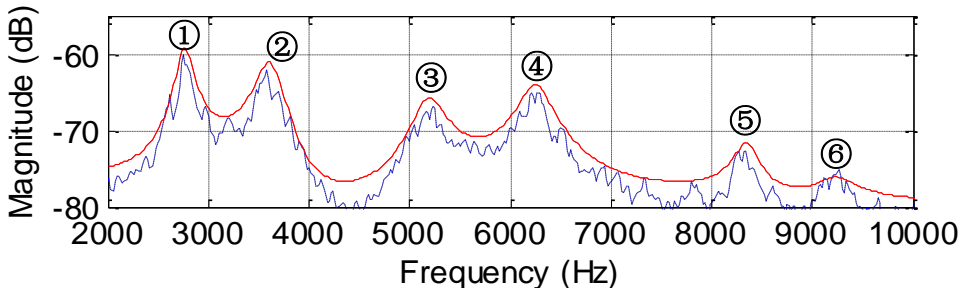

(b) After 400 impacts

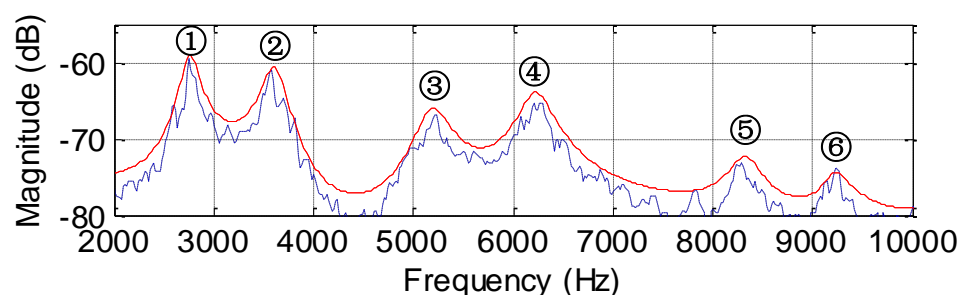

(c) After 700 impacts

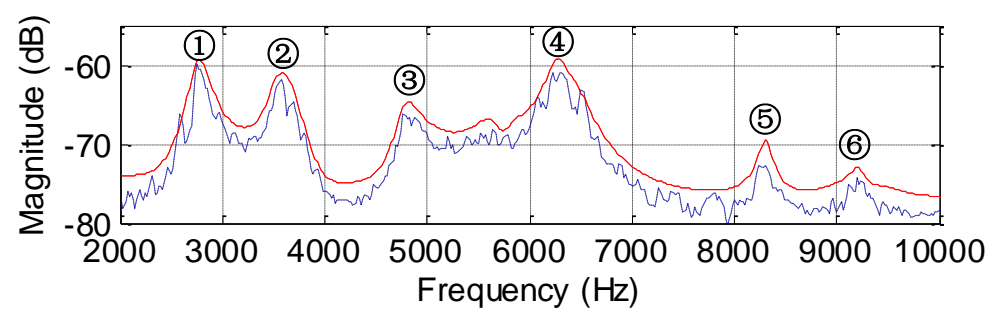

(d) After 1100 impacts

Figure 9. Comparison of estimated and measured FRFs. 


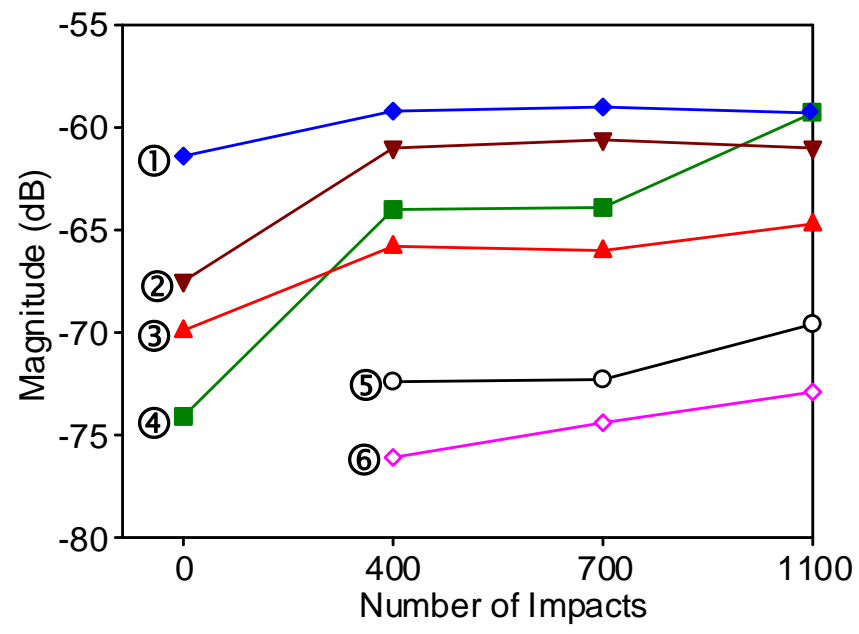

Figure 10. Magnitudes of identified modes

Table II. Magnitude change of the identified mode(4)

\begin{tabular}{|c|c|c|c|c|}
\hline \multirow{2}{*}{ Mode (4) } & \multicolumn{4}{|c|}{ Number of Impacts } \\
\cline { 2 - 5 } & 0 & 400 & 700 & 1,100 \\
\hline Magnitude & -74.1 & -64.0 & -63.9 & -59.3 \\
\hline Increase (dB) & - & 10.1 & 10.2 & 14.8 \\
\hline
\end{tabular}

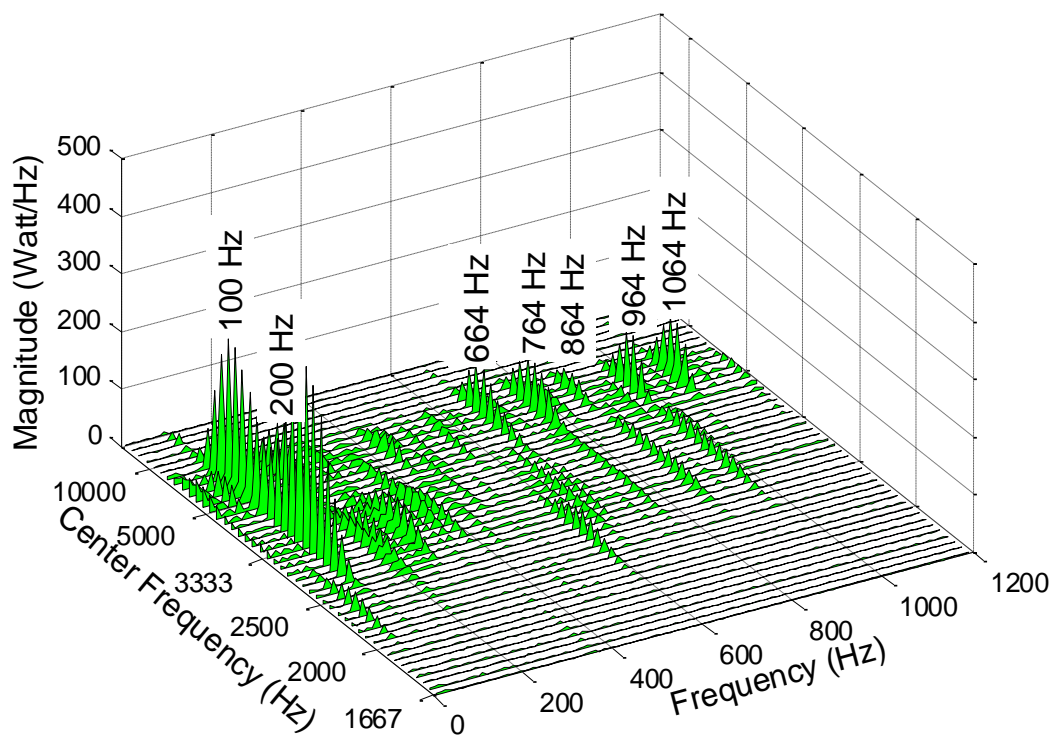

Figure 11. Wavelet envelope spectrum of the vibration signal measured after 1,100 impacts. 


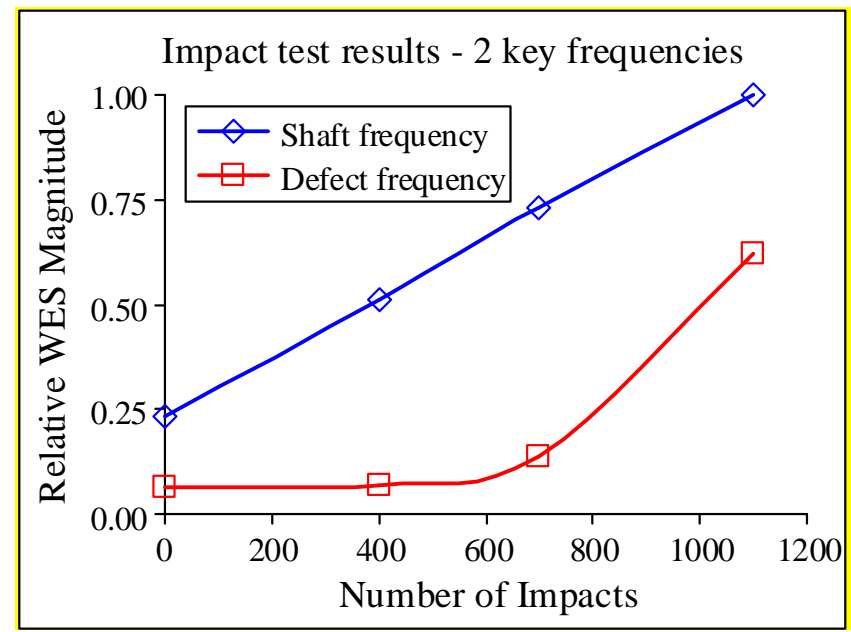

\section{Figure 12. Effects of the accumulated impacts on spindle characteristics}

To specify the effect of static loading on the spindle dynamics, the natural frequencies for four loading conditions were extracted under $126 \mathrm{rad} / \mathrm{s}(1,200 \mathrm{rpm})$ speed. The data sets were collected after 700 impacts. It was found that for all six modes identified in Figure 9, the natural frequencies increase as the static load increases. Several examples are given in Table III, illustrating changes of the natural frequencies for modes (1) and (5). Also, similar trends have been identified for other structural modes, suggesting that increased static load has led to increased stiffness of the spindle structure, as reflected in increased natural frequencies. This finding is in agreement with the investigations reported in [10] and [38].

Table III. Effect of static load on spindle natural frequencies

\begin{tabular}{|c|c|c|c|c|}
\hline \multirow{2}{*}{ Mode } & \multicolumn{4}{|c|}{ Static Load (N) } \\
\cline { 2 - 5 } & 0 & 420 & 840 & 1,260 \\
\hline (1) $(\mathrm{Hz})$ & 2,756 & 2,770 & 2,775 & 2,790 \\
\hline (5) $(\mathrm{Hz})$ & 8,289 & 8,300 & 8,320 & 8,390 \\
\hline
\end{tabular}

The effect of rotational speeds on spindle natural frequencies has also been investigated. Table IV lists the example on the changes of the natural frequencies for mode (1) and (5) under four different speeds. No static load was applied to the spindle. It was noted that the natural frequencies 
decrease with the spindle speed. This phenomenon can be attributed to decreasing stiffness caused by centrifugal forces of the rotating spindle. This is due to the fact that, for a bearing with a fixed outer raceway, centrifugal forces would decrease the contact angle at the outer raceway of the bearing while increasing the contact angle at the inner raceway of the bearing [39]. The changed contact angle difference between outer and inner raceway reduces the stiffness of the bearing. As a result, the natural frequencies will decrease.

Table IV. Effect of speed on spindle natural frequencies

\begin{tabular}{|c|c|c|c|c|}
\hline \multirow{2}{*}{ Mode } & \multicolumn{4}{|c|}{ Speed: rad/s (rpm) } \\
\cline { 2 - 5 } & $\begin{array}{c}126 \\
(1,200)\end{array}$ & $\begin{array}{c}377 \\
(3,600)\end{array}$ & $\begin{array}{c}630 \\
(6,000)\end{array}$ & $\begin{array}{c}879 \\
(8,400)\end{array}$ \\
\hline (1) $(\mathrm{Hz})$ & 2,756 & 2,755 & 2,730 & 2,725 \\
\hline (5) $(\mathrm{Hz})$ & 8,289 & 8,285 & 8,275 & 8,240 \\
\hline
\end{tabular}

\section{Conclusions}

An SSI-based approach has been utilized for in-process modal parameter identification with application to spindle health monitoring. This approach allows for the extraction of spindle modal parameters under actual operating conditions, and does not require any hammer strikes to be applied to the spindle. From experiments conducted on a customized spindle test bed, it was found that the SSI-based approach has returned similar modal identification results to those obtained using the traditional hammer strike method. The in-process applicability of the SSI allowed online identification of changing modal parameters as a result of the accumulated impacts to the spindle. This presents a means for monitoring the structural health of the spindle, and the results obtained provide insight into the working condition of rotating spindles. 


\section{Acknowledgement}

This work was supported by the Smart Machining Systems Program at the National Institute of Standards and Technology (NIST), the National Natural Science Foundation of China (No. 51175080) and the Science and Technology Support Program of Jiangsu Province under grant BE2012740. Collaboration from Kang Lee and Steven Fick at NIST during the experimental study is sincerely appreciated.

\section{References}

[1] Wilson CJ, Bogy DB. An experimental modal analysis technique for miniature structures. ASME Journal of Vibration and Acoustics 1996; 118(1):1-9.

[2] Okubo N, Yoshida Y. Application of modal analysis to machine tool structures. Annals of the CIRP 1982; 31(1):243-246.

[3] Schmitz TL. Predicting high-speed machining dynamics by substructure analysis. Annals of the CIRP 2000; 49(1):303-308.

[4] Budak E, Ertürk A, Özgüven HN. A modeling approach for analysis and improvement of spindle-holder-tool assembly dynamics. Annals of the CIRP 2006; 55(1):369-372.

[5] Smith S, Snyder J. A cutting performance based template for spindle dynamics. Annals of the CIRP 2001; 50(1):259-262.

[6] Park SS, Altintas Y. Dynamic compensation of spindle integrated force sensors with Kalman filter. ASME Journal of Dynamic Systems, Measurement, and Control 2004; 126(3):443-452.

[7] Akturk N. Some characteristic parameters affecting the natural frequency of a rotating shaft supported by defect free ball bearings. Proceedings of the Institute of Mechanical Engineers, Part K: Journal of Multi-body Dynamics 2003; 217(2):145-151.

[8] Alfares MA, Elsharkawy AA. Effects of axial preloading of angular contact ball bearings on the dynamics of a grinding machine spindle system. Journal of Materials Processing Technology 2003; 136 (1-3):48-59.

[9] Zhang L, Yan RQ, Gao R, Lee K. Open architecture software design for online spindle health monitoring. In: Proceedings of the IEEE Measurement and Technology Conference, Warsaw, Poland; May 2007. p. 1-6.

[10] Schmitz TL, Ziegert J, Stanislaus C. A Method for predicting chatter stability for systems with speed-dependent spindle dynamics. Transactions of NAMRI/SME 2004; 32:17-24.

[11] James GH, Carne TG, Lauffer JP, Nord AR. Modal testing using natural excitation. In: Proceedings of the 10th International Modal Analysis Conference, San Diego, CA, USA; February 1992, p. 1209-1216.

[12] James GH, Carne TG, Lauffer JP. The natural excitation technique (Next) for modal parameter 
extraction from operating structures. International Journal of Analytical and Experimental Modal Analysis 1995; 10(4):260-277.

[13] Tasker F, Bosse A, Fisher S. Real-time modal parameter estimation using subspace methods: theory. Mechanical Systems and Signal Processing 1998; 12(6):797-808.

[14] Ren W, Zong Z. Output-only modal parameter identification of civil engineering structures. Structural Engineering and Mechanics 2004; 19(3-4):1-16.

[15] Kim BH, Stubbs N, Park T. A new method to extract modal parameters using output-only responses. Journal of Sound and Vibration 2005; 282(1-2):215-230.

[16] Fan J, Zhang Z, Hua H. Data processing in subspace identification and modal parameter identification of an arch bridge. Mechanical Systems and Signal Processing 2007; 21(4):16741689.

[17] Liu W, Gao W, Sun Y. Application of modal identification methods to spatial structure using field measurement data. ASME Journal of Vibration and Acoustics 2009; 131(3):034503-1-10.

[18] Peeters B, De Roeck G. Reference-based stochastic subspace identification for output-only modal analysis. Mechanical Systems and Signal Processing 1999; 13(6):855-878.

[19] Brincker R, Zhang L, Andersen P. Modal identification from ambient response using frequency domain decomposition. In: Proceedings of the 18th International Modal Analysis Conference, San Antonio, TX, USA; February 2000, p. 625-630.

[20] Ibraham SR. Efficient random decrement computation for identification of ambient responses. In: Proceedings of the 19th International Modal Analysis Conference, Orlando, FL, USA; February 2001, pp 698-703.

[21] Hermans L, Van Der Auweraer H. Model testing and analysis of structures under operational conditions: industrial applications. Mechanical Systems and Signal Processing 1999; 13(2):193-216.

[22] Mevel L, Goursat M. Stochastic subspace-based structural identification and damage detection and localization - application to the Z24 bridge benchmark. Mechanical Systems and Signal Processing 2003; 17(1):143-151.

[23] Sarmadi SAN, Venkatasubramanian V. Electromechanical mode estimation using recursive adaptive stochastic subspace identification. IEEE Transactions on Power Systems 2014; 29(1):349-358.

[24] Basseville M, Basseville M, Benveniste A, Goursat M, Hermans L, Mevel L, Van der Auweraer H. Output-only subspace-based structural identification: from theory to industrial testing practice. ASME Journal of Dynamic Systems, Measurement, and Control 2001; 23(4):668-676.

[25] Mevel L, Benveniste A, Basseville M, Goursat M, Peeters B, Van der Auweraer H, Vecchio A. Input/output versus output-only data processing for structural identification - application to in-flight data analysis. Journal of Sound and vibration 2006; 295(3-5):531-552.

[26] Mrabet E, Abdelghani M, Ben Kahla N. A new criterion for the stabilization diagram used with stochastic subspace identification methods: an application to an aircraft skeleton. Shock and Vibration 2014; 409298.

[27] Schedligski C, Lüscher M. Application of classical and output-only modal analysis to a laser cutting machine. In: Proceedings of International Conference on Noise and Vibration 
Engineering, Leuven, Belgium; September 2002, p. 1273-1279.

[28] Kushnir E. Application of operational modal analysis to a machine tool testing. In: Proceeding of ASME International Mechanical Engineering Congress and Exposition, Anaheim, CA, USA; November 2004, p. 57-62.

[29] Li B, Cai H, Mao XY, Huang JB, Luo B. Estimation of CNC machine-tool dynamic parameters based on random cutting excitation through operational modal analysis. International Journal of Machine Tools \& Manufacture 2013; 71:26-40.

[30] Ozbek M, Meng F, Rixen DJ. Challenges in testing and monitoring the in-operation vibration characteristics of wind turbines. Mechanical Systems and Signal Processing 2013; 41:649-666.

[31] Haeckell MW, Rolfes R. Monitoring a 5 MW offshore wind energy converter-Condition parameters and triangulation based extraction of modal parameters. Mechanical Systems and Signal Processing 2013; 40(1):322-343.

[32] Dong XF, Lian JJ, Yang M, Wang HJ. Operational modal identification of offshore wind turbine structure based on modified stochastic subspace identification method considering harmonic interference. Journal of Renewable and Sustainable Energy 2014; 6(3):033128.

[33] Brendelson JC, Dhingra AK. Stochastic subspace identification applied to the weave mode of motorcycles. ASME Journal of Dynamic Systems Measurement and Control 2013; 135(2):021019.

[34] Peeters B, De Roeck G. Stochastic system identification for operational modal analysis: a review. ASME Journal of Dynamic Systems, Measurement, and Control 2001; 123(4):659-667.

[35] Ibrahim SR, Brincker R, Asmussen JC. Modal parameter identification from responses of general unknown random inputs. In: Proceedings of the 14th International Modal Analysis Conference, Dearborn, MI, USA; February 1996, p. 446-452.

[36] Richardson MH, Formenti DL. Parameter estimation from frequency response measurements using rational fraction polynomials. In: Proceedings of the First International Modal Analysis Conference, Orlando, FL, USA; November 1982, p. 167-181.

[37] Zhang L, Gao R, Lee K. Spindle health diagnosis based on analytic wavelet enveloping, IEEE Transactions on Instrumentation and Measurement 2006; 55(5):1850-1858.

[38] Altintas Y, Cao Y. Virtual design and optimization of machine tool spindles. Annals of the CIRP 2005; 54(1):379-382.

[39] Abele E, Fiedler U. Creating stability lobe diagrams during milling. Annals of the CIRP 2004; 53(1):309-313. 